\title{
Love Waves in a Heterogeneous Orthotropic Layer Under Initial Stress Overlying a Gravitating Porous Half-Space
}

\author{
ANUP SAHA*, SANTIMOY KUNDU, SHISHIR GUPTA and PRAMOD KUMAR VAISHNAV \\ Department of Applied Mathematics, Indian School of Mines, Dhanbad 826 004, India
}

(Received on 02 June 2015; Revised on 14 July 2015; Accepted on 16 July 2015)

\begin{abstract}
In this paper, Love waves in a heterogeneous orthotropic layer under changeable initial stress over a gravitating porous halfspace have been investigated. The dispersion equation of Love waves has been deducted in a closed form by means of the method of variable separation. The frequency equation shows that heterogeneity, initial stress, ratio of directional rigidities as well as gravity and porosity of the porous half-space have notable effect on the propagation of Love waves. The velocity of Love waves has been computed for several cases. It has also been observed that for a homogeneous orthotropic layer over a homogeneous orthotropic half-space, the velocity equation obtained is in agreement with the corresponding result.
\end{abstract}

Keywords: Love Waves; Gravity; Initial Stress; Orthotropic Medium; Porosity; Heterogeneity

\section{Introduction}

Since the structure of the Earth's interior cannot be studied directly, Earth scientists use seismic waves to study the deeper layers of the Earth. Most of the information on the constitution of the Earth's interior has been obtained from studies of seismic body waves. Most hypotheses about the internal structure of the Earth can be developed by studying seismic waves that travel through the Earth and are measured at seismic stations.

There are several types of seismic waves and they all move in different ways. One of these is surface wave which can only move along the surface of the planet like ripples on water. The study of surface waves in a half-space is important due to its possible applications in geophysical prospecting and in understanding the cause and damage due to earthquake. Surface waves carry the greater amount of energy from shallow shocks and are the primary cause of destruction that can result from earthquakes. Quite a good amount of information about the propagation of seismic waves in layered media is contained in the well-known book by Ewinget al. (1957). A large number of papers has been published in different journals since this book was published. Propagation of Love waves in homogeneous micropolar isotropic elastic media consisting of a layer of finite thickness lying over a semi-infinite media has been considered by Midya (2004). Manna et al. (2013) showed that the phase velocities decrease with the increase of inhomogeneity parameters.

The development of initial stress in the medium is due to many reasons, for example the difference of temperature, process of quenching, gravity variation, creep, differential external forces, etc. These stresses have significant influence on the elastic waves produced by earthquake explosions or impacts. The Earth may be assumed as elastic solid layered medium under high initial stress. It is therefore of much interest to study the influences of these stresses on the propagation of Love waves. Dey and Dutta (1992) discussed torsional wave propagation in an initially stressed cylinder. Abd-Alla and Ahmed (1999)

*Authorfor Correspondence: E-mail: sahaanup1989@gmail.com 
showed the effect of non-homogeneity of the orthotropic media as well as the changeable initial stress on the propagation of Love waves. Ahmed and Abo-Dahab (2010) investigated Love waves in an orthotropic Granular layer under initial stress overlying a semi-infinite Granular medium. AbdAlla et al. (2013a) studied Love waves in a nonhomogeneous orthotropic magneto-elastic layer under initial stress overlying a semi-infinite medium. Gupta et al. (2013a) attempted to show the effect of initial stress on the propagation of Love wave when upper boundary plane is completely free surface. Kundu et al. (2014a) observed the influence of fibrereinforced parameters and initial stress on phase velocity of Love waves.

Recently, the study of wave propagation in porous medium has gained prime interest. The propagation of Love wave in a heterogeneous medium over a semi-infinite porous medium has great importance in earthquake engineering and seismology on account of the occurrence of porosity and heterogeneity in the crust of the Earth as the Earth is supposed to be made up of different layers. Naturally occurring media is often porous and is liquid filled. The size of pores is assumed to be small and macroscopically speaking, their average distribution is uniform. Various problems of waves and vibrations based on these theories of elasticity have been attempted by the researchers and appeared in the open literature. Chattopadhyay and De (1983) studied Love type waves in a porous layer with irregular interface. Longitudinal and shear waves in void medium has been discussed by Dey and Gupta (1987). Propagation of Love wave in an initially stressed fluidsaturated porous layer with an irregular boundary sandwiched between two isotropic half-spaces was studied by Chattaraj et al. (2013b) and it was observed that phase velocity decreases with an increase in the dimensionless wave number for a fixed value of the parameter of irregularity. Gupta et al. (2013b) used an analytical approach to investigate Love wave propagation in an anisotropic porous layer under the effect of rigid boundary and showed the effect of porosity, anisotropy, non-homogeneity and initial stress on the phase velocity of Love wave. Kundu et al. (2013) found that the phase velocity of Love waves is considerably influenced by rigidity, porosity and anisotropy of the porous layer, inhomogeneity of the half-space and prestressing present in the media, the layer and the half-space. The existence of Love wave propagation in an initially stressed homogeneous layer over a porous half-space with irregular boundary surface was investigated by Kundu et al. (2014b).

The Earth is a gravitating medium. Presence of gravity field effects the propagation of seismic waves. The acceleration due to gravity has a great importance in analyzing the dynamics and static problems of the Earth. Dey et al. (1998) introduced the constitutive equation for the propagation of torsional surface waves in dry sandy medium under gravity. Ghorai et al. (2010) considered a mathematical model for the propagation of Love waves in a fluid-saturated porous layer under a rigid boundary and lying over an elastic half-space under gravity. Abd-Allat al. (2012) established a mathematical expression about the propagation of Rayleigh waves in a rotating orthotropic material elastic half-space under initial stress and gravity. Chattaraj and Samal (2013a) established that the transverse and longitudinal rigidity of fibre-reinforced material, gravity and porosity of the half-space have significant effect on the propagation of Love waves. Abd-Allaet al. (2013b) showed the effect of gravity, anisotropy and parameters for fibre-reinforcement of the material medium on surface waves.

The propagation of elastic waves in orthotropic media is fundamentally different from their propagation in isotropic media. So the study of surface waves in orthotropic layered media has become of prime interest to seismologist and geophysicist. The study of generations and propagation of waves in layered orthotropic media with various geometrical configurations is highly important in Geophysics, Seismology, Acoustics and Electromagnetism. However, no attempt has been made to study the propagation of Love wave in a heterogeneous orthotropic layer under changeable initial stress over a porous half-space under gravity.

In the present paper, the heterogeneity of the layer is taken as, $P=P_{0}(1+\sin \alpha z), \rho=\rho_{0}(1+\sin \alpha z)$, $Q_{i}=a_{i}(1+\sin \alpha z)$ where $P$ is initial stress, $\rho$ is density, 
$Q_{i}$ are shear moduli, $\alpha$ is a constant having dimension that is inverse of length. It is observed that Love waves exist in the assumed media. Using variable separable method, the dispersion equation has been obtained in a closed form and the results have been discussed. The study reveals that the heterogeneity as well as the initial stress, porosity and gravity have remarkable effect on the propagation of Love waves.

\section{Statement of the Problem}

Consider a heterogeneous orthotropic layer of finite thickness $H$ over an anisotropic porous halfspace under gravity under initial stress $P$. We assume the upper half-space of the orthotropic layer is free and horizontal. The $z$-axis is taken vertically downward in the porous half-space and the $x$-axis is considered parallel to the layer in the direction of wave propagation. The origin is taken at a depth $H$ below the upper surface of orthotropic layer. Geometrical picture of the problem is shown in Fig. 1.

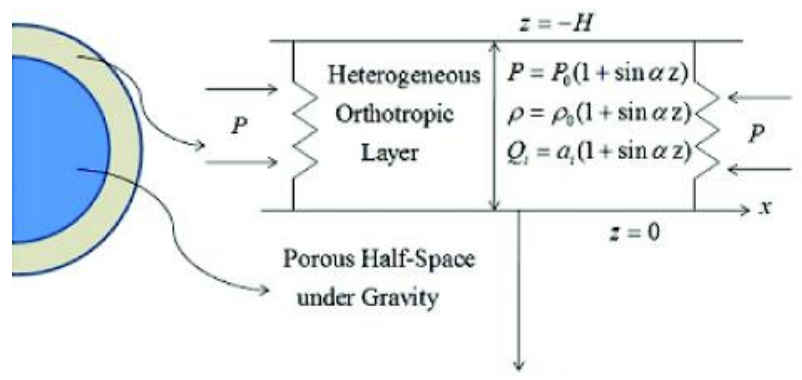

Fig. 1: Geometry of the problem

The following variations in initial stress, density and shear moduli in the layer are taken into account:

$$
\left.\begin{array}{l}
P=P_{0}(1+\sin \alpha \mathrm{z}), \\
\rho=\rho_{0}(1+\sin \alpha \mathrm{z}), \\
Q_{i}=a_{i}(1+\sin \alpha \mathrm{z}),
\end{array}\right\}
$$

Where, $P$ is initial stress, $O$ is density, $Q_{i}$ are shear moduli, $\alpha$ is a constant having dimension that is inverse of length. Here $P_{0}, o_{0}, a_{i}$ are the values of $P, o$ and $Q_{i}$ at $z=0$.

\section{Solution for Heterogeneous Orthotropic Layer}

The dynamical equations of motion in the absence of body force under initial stress $\left(\sigma_{11}=-\mathrm{P}\right)$ are

$$
\left.\begin{array}{l}
\frac{\partial \sigma_{11}}{\partial x}+\frac{\partial \sigma_{12}}{\partial y}+\frac{\partial \sigma_{13}}{\partial z}-P\left(\frac{\partial w_{z}}{\partial y}-\frac{\partial w_{y}}{\partial z}\right)=\rho \frac{\partial^{2} u}{\partial t^{2}} \\
\frac{\partial \sigma_{12}}{\partial x}+\frac{\partial \sigma_{22}}{\partial y}+\frac{\partial \sigma_{23}}{\partial z}-P\left(\frac{\partial w_{z}}{\partial x}\right)=\rho \frac{\partial^{2} v}{\partial t^{2}} \\
\frac{\partial \sigma_{13}}{\partial x}+\frac{\partial \sigma_{23}}{\partial y}+\frac{\partial \sigma_{33}}{\partial z}-P\left(\frac{\partial w_{y}}{\partial x}\right)=\rho \frac{\partial^{2} w}{\partial t^{2}}
\end{array}\right\}
$$

Where, $\sigma_{i j}(i, j=1,2,3)$ are the incremental stress components; $u, v, w$ are the components of the displacement vector along $x, y, z$ directions respectively; $w_{x}, w_{y}, w_{z}$ are the angular displacement vectors given by

$$
\begin{aligned}
& w_{x}=\frac{1}{2}\left(\frac{\partial w}{\partial y}-\frac{\partial v}{\partial z}\right), w_{y}=\frac{1}{2}\left(\frac{\partial u}{\partial z}-\frac{\partial w}{\partial x}\right) \\
& w_{z}=\frac{1}{2}\left(\frac{\partial v}{\partial x}-\frac{\partial u}{\partial y}\right)
\end{aligned}
$$

The relations between the strain and incremental stress components are:

$$
\left.\begin{array}{l}
\sigma_{11}=B_{11} e_{x x}+B_{12} e_{y y}+B_{13} e_{z z}, \\
\sigma_{11}=B_{21} e_{x x}+B_{22} e_{y y}+B_{23} e_{z z}, \\
\sigma_{11}=B_{31} e_{x x}+B_{32} e_{y y}+B_{33} e_{z z}, \\
\sigma_{12}=2 Q_{3} e_{x y}, \\
\sigma_{23}=2 Q_{1} e_{y z}, \\
\sigma_{31}=2 Q_{2} e_{z x},
\end{array}\right\}
$$

Where, $B_{i j}$ are the incremental normal elastic coefficients and $Q_{i}$ are the shear moduli.

For the propagation of Love waves along $x$-axis, we have

$$
u=0, w=0, v=v(\mathrm{x}, \mathrm{z}, \mathrm{t})
$$

The strain-displacement relations are 


$$
\begin{aligned}
& e_{x y}=\frac{1}{2}\left(\frac{\partial u}{\partial y}+\frac{\partial v}{\partial x}\right), e_{y z}=\frac{1}{2}\left(\frac{\partial v}{\partial z}+\frac{\partial w}{\partial y}\right) \\
& e_{z x}=\frac{1}{2}\left(\frac{\partial u}{\partial z}+\frac{\partial w}{\partial x}\right)
\end{aligned}
$$

Using the relationships (3.2), (3.3), (3.4) and (3.5), the equation of motion given by (3.1) becomes

$$
\begin{aligned}
Q_{1} \frac{\partial^{2} v}{\partial z^{2}} & +\left(Q_{3}-\frac{P}{2}\right) \frac{\partial^{2} v}{\partial x^{2}}+\frac{\partial Q_{3}}{\partial x} \frac{\partial v}{\partial x} \\
+ & \frac{\partial Q_{1}}{\partial z} \frac{\partial v}{\partial v}=\rho \frac{\partial^{2} v}{\partial t^{2}} .
\end{aligned}
$$

For a wave propagation along $x$-direction, we may assume

$$
v=V(\mathrm{z}) e^{i k(\mathrm{x}-\mathrm{ct})},
$$

where $k$ is wave number, $\omega(=k c)$ is angular frequency, $c$ is the speed of simple harmonic waves of wavelength $\frac{2 \pi}{k}$ travelling forward.

Equation (3.6) with the help of equation (3.7) becomes

$$
\begin{aligned}
Q_{1} \frac{d^{2} V}{d z^{2}} & +\frac{\partial Q_{1}}{\partial z} \frac{d v}{d z}+i k \frac{\partial Q_{1}}{\partial x} V \\
& +k^{2}\left(\rho c^{2}+\frac{P}{2}-Q_{3}\right) V=0
\end{aligned}
$$

Now using the transformations (2.1), the above equation changes to

$$
\begin{aligned}
\frac{d^{2} V}{d z^{2}}+ & \frac{\alpha \cos (\alpha \mathrm{z})}{1+\sin (\alpha \mathrm{z})} \frac{d V}{d z} \\
& +k^{2}\left(\frac{c^{2}}{c_{1}^{2}}+\frac{P_{0}-2 a_{3}}{2 a_{1}}\right) V=0,
\end{aligned}
$$

where $c_{1}=\sqrt{\frac{a_{1}}{\rho_{0}}}$ is the shear wave velocity in the upper heterogeneous orthotropic layer. On substituting $V(\mathrm{z})=\frac{\varphi(\mathrm{z})}{\sqrt{1+\sin (\alpha \mathrm{z})}}$ into equation (3.9) to eliminate the $\frac{d V}{d z}$ term we get,

$$
\frac{d^{2} \varphi}{d z^{2}}+m^{2} \varphi=0
$$

where $m^{2}=k^{2}\left(\frac{\alpha^{2}}{4 k^{2}}+\frac{c^{2}}{c_{1}^{2}}+\frac{P_{0}-2 a_{3}}{2 a_{1}}\right)$

The solution of (3.10) is

$$
\varphi(\mathrm{z})=\mathrm{A}_{1} \cos (\mathrm{mz})+\mathrm{A}_{2} \sin (\mathrm{mz}),
$$

where $A_{1}$ and $A_{2}$ are arbitrary constants.

Hence the displacement component in the upper heterogeneous orthotropic layer is

$$
v=\frac{\mathrm{A}_{1} \cos (\mathrm{mz})+\mathrm{A}_{2} \sin (\mathrm{mz})}{\sqrt{1+\sin (\alpha \mathrm{z})}} e^{i k(\mathrm{x}-\mathrm{ct})} .
$$

\section{Solution for Porous Half-Space}

If $\left(u^{\prime}, v^{\prime}, w^{\prime}\right)$ and $\left(\mathrm{U}^{\prime}, \mathrm{V}^{\prime}, \mathrm{W}^{\prime}\right)$ are the displacements of solid and liquid parts of a porous aggregate respectively, then for the propagation of Love wave along $x$ axis is given by

$$
\left.\begin{array}{l}
u^{\prime}=0, \mathrm{w}^{\prime}=0, \mathrm{v}^{\prime}=\mathrm{v}^{\prime}(\mathrm{x}, \mathrm{z}, \mathrm{t}) \\
\mathrm{U}^{\prime}=0, \mathrm{~W}^{\prime}=0, \mathrm{~V}^{\prime}=\mathrm{V}^{\prime}(\mathrm{x}, \mathrm{z}, \mathrm{t})
\end{array}\right\}
$$

Dynamical equation of motion for anisotropic porous half-space under gravity in the absence of body force is (Biot, 1965).

$$
\begin{aligned}
& \frac{\partial \tau_{12}}{\partial x}+\frac{\partial \tau_{23}}{\partial z}-d^{\prime} g w_{23}^{\prime}-d^{\prime} g z \frac{\partial w_{23}^{\prime}}{\partial z} \\
& +d^{\prime} g z \frac{\partial w_{12}^{\prime}}{\partial x}=\frac{\partial^{2}}{\partial t^{2}}\left(\rho_{11} v^{\prime}+\rho_{12} V^{\prime}\right),
\end{aligned}
$$

and 


$$
\frac{\partial \tau}{\partial y}=\frac{\partial^{2}}{\partial t^{2}}\left(\rho_{12} v^{\prime}+\rho_{22} V^{\prime}\right)
$$

where $\tau_{i j}$ and $\tau$ are incremental stress components of solid and stress in liquid respectively; $\left(u^{\prime}, v^{\prime}, w^{\prime}\right)$ are components of the displacement vector of the solid; $\left(\mathrm{U}^{\prime}, \mathrm{V}^{\prime}, \mathrm{W}^{\prime}\right)$ are components of the displacement vector of the liquid; and $w_{i j}^{\prime}$ are the angular components defined as

$$
w_{12}^{\prime}=-\frac{1}{2} \frac{\partial v^{\prime}}{\partial x}, \quad w_{23}^{\prime}=\frac{1}{2} \frac{\partial v^{\prime}}{\partial z}
$$

The stress-displacement relations are

$$
\tau_{12}=N \frac{\partial v^{\prime}}{\partial x}, \quad \tau_{23}=L \frac{\partial v^{\prime}}{\partial z}
$$

where $N$ and $L$ are shear moduli of anisotropic halfspace along $x$ and $z$ directions respectively.

Under the assumption that there is no relative motion between liquid and solid in porous structure, the mass coefficients $o_{11}, O_{12}$ and $O_{22}$ are related to the densities $\rho^{\prime}, o_{s}$ and $o_{f}$ of the solid-liquid aggregate, solid and liquid respectively (Biot, 1956) by

$$
\begin{aligned}
& \rho_{11}+\rho_{12}=(1-f) \rho_{s}, \\
& \rho_{12}+\rho_{22}=f \rho_{f}, \\
& \rho^{\prime}+\rho_{s}+f\left(\rho_{f}-\rho_{s}\right),
\end{aligned}
$$

where $f$ is porosity of half-space.

These mass coefficients also obey the following inequalities

$\rho_{11}>0, \quad \rho_{22}>0, \quad \rho_{12}<0, \quad \rho_{11} \rho_{22}-\rho_{12}^{2}>0$.

Eliminating the displacement component $\mathrm{V}^{\prime}$ from equations (4.2) and (4.3) we get,

$$
\begin{gathered}
\frac{\partial \tau_{12}}{\partial x}+\frac{\partial \tau_{23}}{\partial z}-d^{\prime} g w_{23}^{\prime}-d^{\prime} g z \frac{\partial w_{23}^{\prime}}{\partial z} \\
+d^{\prime} g z \frac{\partial w_{12}^{\prime}}{\partial x}=d^{\prime} \frac{\partial^{2} v^{\prime}}{\partial t^{2}}
\end{gathered}
$$

where

$$
d^{\prime}=\rho_{11}-\frac{\rho_{12}^{2}}{\rho_{22}}
$$

Now using the relations (4.4) and (4.5) in equation (4.6), one gets

$$
\begin{array}{r}
\left(N-\frac{d^{\prime} g z}{2}\right) \frac{\partial^{2} v^{\prime}}{\partial x^{2}}+\left(L-\frac{d^{\prime} g z}{2}\right) \frac{\partial^{2} v^{\prime}}{\partial z^{2}} \\
-\frac{d^{\prime} g}{2} \frac{\partial v^{\prime}}{\partial z}=d^{\prime} \frac{\partial^{2} v^{\prime}}{\partial t^{2}}
\end{array}
$$

Again we consider the harmonic solution of equation (4.7) of the form

$$
v^{\prime}=V(\mathrm{z}) \mathrm{e}^{i k(\mathrm{x}-\mathrm{ct})}
$$

where $V(z)$ is the solution of

$$
\frac{d^{2} V}{d z^{2}}-\frac{G d k}{2\left(1-\frac{G d k z}{2}\right)} \frac{d V}{d z}-k^{2}
$$

$$
\left[\frac{\left(\frac{N}{L}-\frac{G d k z}{2}\right)-\frac{d c^{2}}{c^{2}}}{1-\frac{G d k z}{2}}\right] V=0
$$

In the above, $c_{2}=\sqrt{\frac{L}{\rho^{\prime}}}$ is the shear wave velocity in the porous half-space; $G=\frac{\rho^{\prime} g}{L k}$ is the Biot's gravity parameter; $d=\frac{d^{\prime}}{\rho^{\prime}}=\gamma_{11}-\frac{\gamma_{12}^{2}}{\gamma_{22}}$, where $\gamma_{11}=\frac{\rho_{11}}{\rho^{\prime}}, \quad \gamma_{12}=\frac{\rho_{12}}{\rho^{\prime}}, \quad \gamma_{22}=\frac{\rho_{22}}{\rho^{\prime}}$ are the non-dimensionless parameters for the material of the porous half-space as obtained by Biot. 
Substituting $V(\mathrm{z})=\frac{\psi(\mathrm{z})}{\sqrt{1-\frac{G d k z}{2}}}$ into equation

(4.9) to eliminate the term $\frac{d V}{d z}$ we obtain,

$$
\begin{aligned}
\frac{d^{2} \psi}{d z^{2}}+k^{2} & {\left[\frac{G^{2} d^{2}}{16\left(1-\frac{G d k z}{2}\right)^{2}}-\frac{\left(\frac{N}{L}-\frac{G d k z}{2}\right)-\frac{d c^{2}}{c^{2}}{ }_{2}}{1-\frac{G d k z}{2}}\right] } \\
\psi & =0
\end{aligned}
$$

Using $\psi(\mathrm{z})=\mathrm{g}(\eta)$ in the above equation where $\eta=\frac{4}{G d}\left(1-\frac{G d k z}{2}\right)$ one gets

$$
\frac{d^{2} g}{d \eta^{2}}+\left[-\frac{1}{4}+\frac{1}{4 \eta^{2}}+\frac{R}{\eta}\right] g=0,
$$

where $R=\frac{1}{G}\left\{\left(1-\frac{N}{L}\right) \frac{1}{d}+\frac{c^{2}}{c^{2}}{ }_{2}\right\}$.

The solution of equation (4.11) satisfying the boundary condition $v^{\prime} \rightarrow 0$ as $z \rightarrow \infty$, i.e., $g(\eta) \rightarrow 0$ as $\eta \rightarrow-\infty$ may be taken as

$$
\mathrm{g}(\eta)=\mathrm{A}_{3} W_{-R, 0}(-\eta)
$$

where $W_{-R, 0}(-\eta)$ is the Whittaker function (Whittaker and Watson, 1990).

Expanding the Whittaker function up to linear terms, we get the displacement for Love wave in the lower porous half-space as

$$
\begin{aligned}
& v^{\prime}=A_{3}\left(-\frac{4}{G d}\right)^{-R} e^{\frac{2}{G d}\left(1-\frac{G d k z}{2}\right)}\left(1-\frac{G d k z}{2}\right)^{-R-\frac{1}{2}} \\
& \left\{1+\frac{G d}{4} \frac{\left(R+\frac{1}{2}\right)^{2}}{1-\frac{G d k z}{2}} e^{i k(x-c t) .}\right.
\end{aligned}
$$

\section{Boundary Conditions}

The following boundary conditions must be satisfied: (i) At the free surface $z=-H$, the stress is vanishing so that

$$
a_{1} \frac{\partial v}{\partial z}=0 \text { at } z=-H
$$

(ii) The continuity of the displacement component requires

$$
v=v^{\prime} \text { at } z=0
$$

(iii) At the interface $z=0$, the continuity of stress component requires that

$$
a_{1} \frac{\partial v}{\partial z}=L \frac{\partial v^{\prime}}{\partial z} \text { at } z=0
$$

Using equations (3.11) and (4.12) in the above boundary conditions, the following equations are obtained

$$
\begin{gathered}
A_{1}\left[m \sin (\mathrm{mH})-\frac{\alpha \cos (\alpha \mathrm{H}) \cos (\mathrm{mH})}{2(1-\sin (\alpha \mathrm{H}))}\right] \\
+A_{2}\left[m \cos (\mathrm{mH})+\frac{\alpha \cos (\alpha \mathrm{H}) \cos (\mathrm{mH})}{2(1-\sin (\alpha \mathrm{H}))}\right]=0 \\
\mathrm{~A}_{1}=A_{3} S_{1}, \\
A_{1}\left(-\frac{\alpha}{2}\right)+A_{2}(\mathrm{~m})=\mathrm{A}_{3}\left(\frac{L S_{2}}{a_{1}}\right)
\end{gathered}
$$

Where,

$$
\begin{aligned}
& S_{1}=\left(-\frac{4}{G d}\right)^{-R} e^{\frac{2}{G d}}\left\{1+\frac{G d}{4}\left(R+\frac{1}{2}\right)^{2}\right\}, \\
& S_{2}=k\left(-\frac{4}{G d}\right)^{-R} e^{\frac{2}{G d}}
\end{aligned}
$$




$$
\left[\left\{1+\frac{G d}{4}\left(R+\frac{1}{2}\right)^{2}\right\}\left\{\frac{G d}{2}\left(R+\frac{1}{2}\right)-1\right\}+\frac{G^{2} d^{2}}{8}\left(R+\frac{1}{2}\right)^{2}\right]
$$

Eliminating the arbitrary constants $A_{1}, A_{2}$ and $A_{3}$ from the above three equations we get

$\tan \left[k H \sqrt{\left(\frac{\alpha}{2 k}\right)^{2}+\frac{c^{2}}{c^{2}{ }_{1}}+\frac{P_{0}-2 a_{3}}{2 a_{1}}}\right]=\frac{\left[\left(\frac{\alpha}{2 k}\right) \frac{\cos (\alpha \mathrm{H})}{1-\sin (\alpha \mathrm{H})}-\left(\frac{L}{a_{1}} \frac{S_{2}}{S_{1} k}+\frac{\alpha}{2 k}\right)\right] \sqrt{\left(\frac{\alpha}{2 k}\right)^{2}+\frac{c^{2}}{c^{2}}+\frac{P_{0}-2 a_{3}}{2 a_{1}}}}{\left(\frac{\alpha}{2 k}\right)^{2}+\frac{c^{2}}{c^{2}{ }_{1}}+\frac{P_{0}-2 a_{3}}{2 a_{1}}+\left(\frac{\alpha}{2 k}\right) \frac{\cos (\alpha \mathrm{H})}{1-\sin (\alpha \mathrm{H})}\left(\frac{L}{a_{1}} \frac{S_{2}}{S_{1} k}+\frac{\alpha}{2 k}\right)}$.

Where,

$$
\frac{S_{2}}{S_{1} k}=\frac{G d}{2}\left(R+\frac{1}{2}\right)-1+\frac{\frac{G^{2} d^{2}}{8}\left(R+\frac{1}{2}\right)^{2}}{1+\frac{G d}{4}\left(R+\frac{1}{2}\right)^{2}} .
$$

Equation (5.7) gives the phase velocity of Love wave in a heterogeneous orthotropic layer under initial stress over a porous half-space under gravity.

\section{Particular Cases}

If the lower half-space is non-porous, i.e., $f \rightarrow 0$, then $\rho_{s} \rightarrow \rho^{\prime}$ which implies $\gamma_{11}+\gamma_{12} \rightarrow 1$ and $\gamma_{12}+\gamma_{22} \rightarrow 0$ and so $d=\gamma_{11}-\frac{\gamma_{12}^{2}}{\gamma_{22}} \rightarrow 1$

Again if $f \rightarrow 1$, then $\rho_{f} \rightarrow \rho^{\prime}$ and the half-space becomes fluid. In this case $d \rightarrow 0$.

Therefore for a non-porous solid $d=1$, for fluid $d=0$ and for porous solid filled with liquid $0<d<1$.

Case I: In the absence of initial stress in the layer, i.e., when $P_{0}=0$, the dispersion equation (5.7) reduces to

$$
\tan \left[k H \sqrt{\left(\frac{\alpha}{2 k}\right)^{2}+\frac{c^{2}}{c^{2}{ }_{1}}-\frac{a_{3}}{a_{1}}}\right]=\frac{\left[\left(\frac{\alpha}{2 k}\right) \frac{\cos (\alpha \mathrm{H})}{1-\sin (\alpha \mathrm{H})}-\left(\frac{L}{a_{1}} \frac{S_{2}}{S_{1} k}+\frac{\alpha}{2 k}\right)\right] \sqrt{\left(\frac{\alpha}{2 k}\right)^{2}+\frac{c^{2}}{c^{2}}-\frac{a_{3}}{a_{1}}}}{\left(\frac{\alpha}{2 k}\right)^{2}+\frac{c^{2}}{c^{2}{ }_{1}}-\frac{a_{3}}{a_{1}}+\left(\frac{\alpha}{2 k}\right) \frac{\cos (\alpha \mathrm{H})}{1-\sin (\alpha \mathrm{H})}\left(\frac{L}{a_{1}} \frac{S_{2}}{S_{1} k}+\frac{\alpha}{2 k}\right)} .
$$

This is the dispersion equation of Love wave in a heterogeneous orthotropic layer in the absence of initial stress over a porous half-space under gravity. 
Case II: If the upper layer is isotropic and free from initial stress, i.e., $a_{1}=a_{3}=\mu_{1}, P_{0}=0$ the dispersion equation of Love wave becomes

$$
\tan \left[k H \sqrt{\left(\frac{\alpha}{2 k}\right)^{2}+\frac{c^{2}}{c^{2}{ }_{1}}-1}\right]=\frac{\left[\left(\frac{\alpha}{2 k}\right) \frac{\cos (\alpha \mathrm{H})}{1-\sin (\alpha \mathrm{H})}-\left(\frac{L}{\mu_{1}} \frac{S_{2}}{S_{1} k}+\frac{\alpha}{2 k}\right)\right] \sqrt{\left(\frac{\alpha}{2 k}\right)^{2}+\frac{c^{2}}{c^{2}}-1}}{\left(\frac{\alpha}{2 k}\right)^{2}+\frac{c^{2}}{c^{2}{ }_{1}}-1+\left(\frac{\alpha}{2 k}\right) \frac{\cos (\alpha \mathrm{H})}{1-\sin (\alpha \mathrm{H})}\left(\frac{L}{\mu_{1}} \frac{S_{2}}{S_{1} k}+\frac{\alpha}{2 k}\right)} .
$$

Case III: When $\alpha \rightarrow 0$, i.e., the upper layer is homogeneous, equation (5.7) takes the form

$$
\tan \left[k H \sqrt{\frac{c^{2}}{c^{2}}+\frac{P_{0}-2 a_{3}}{2 a_{1}}}\right]=-\frac{\frac{L}{a_{1}} \frac{S_{2}}{S_{1} k}}{\sqrt{\frac{c^{2}}{c^{2}}+\frac{P_{0}-2 a_{3}}{2 a_{1}}}}
$$

which is the dispersion equation of Love wave in a homogeneous orthotropic layer under initial stress over a porous half-space under gravity.

Case IV: When $G=0$, i.e., the half-space is free from gravity, the dispersion equation changes into

$$
\tan \left[k H \sqrt{\left(\frac{\alpha}{2 k}\right)^{2}+\frac{c^{2}}{c^{2}{ }_{1}}+\frac{P_{0}-2 a_{3}}{2 a_{1}}}\right]=\frac{\left[\left(\frac{\alpha}{2 k}\right) \frac{\cos (\alpha \mathrm{H})}{1-\sin (\alpha \mathrm{H})}-\left(\frac{L}{a_{1}} S_{3}+\frac{\alpha}{2 k}\right)\right] \sqrt{\left(\frac{\alpha}{2 k}\right)^{2}+\frac{c^{2}}{c^{2}}+\frac{P_{0}-2 a_{3}}{2 a_{1}}}}{\left(\frac{\alpha}{2 k}\right)^{2}+\frac{c^{2}}{c^{2}{ }_{1}}+\frac{P_{0}-2 a_{3}}{2 a_{1}}+\left(\frac{\alpha}{2 k}\right) \frac{\cos (\alpha \mathrm{H})}{1-\sin (\alpha \mathrm{H})}\left(\frac{L}{a_{1}} S_{3}+\frac{\alpha}{2 k}\right)} .
$$

where $S_{3}=\frac{1}{2}\left\{\left(1-\frac{N}{L}+d \frac{c^{2}}{c^{2}}\right)\right\}-1$.

Case V: When $G=0$ and $d \rightarrow 1$, i.e., the lower half-space is non-porous and free from gravity, equation (5.7) becomes

$$
\tan \left[k H \sqrt{\left(\frac{\alpha}{2 k}\right)^{2}+\frac{c^{2}}{c^{2}{ }_{1}}+\frac{P_{0}-2 a_{3}}{2 a_{1}}}\right]=\frac{\left[\left(\frac{\alpha}{2 k}\right) \frac{\cos (\alpha \mathrm{H})}{1-\sin (\alpha \mathrm{H})}-\left(\frac{L}{a_{1}} S_{4}+\frac{\alpha}{2 k}\right)\right] \sqrt{\left(\frac{\alpha}{2 k}\right)^{2}+\frac{c^{2}}{c^{2}}+\frac{P_{0}-2 a_{3}}{2 a_{1}}}}{\left(\frac{\alpha}{2 k}\right)^{2}+\frac{c^{2}}{c^{2}{ }_{1}}+\frac{P_{0}-2 a_{3}}{2 a_{1}}+\left(\frac{\alpha}{2 k}\right) \frac{\cos (\alpha \mathrm{H})}{1-\sin (\alpha \mathrm{H})}\left(\frac{L}{a_{1}} S_{4}+\frac{\alpha}{2 k}\right)}
$$

where $S_{4}=\frac{1}{2}\left\{\left(1-\frac{N}{L}+\frac{c^{2}}{c^{2}{ }_{2}}\right)\right\}-1$ 
Case VI: If the half-space is non-porous, isotropic (i.eV $\left.=L=\mu_{2}\right)$ and free from gravity, the dispersion equation of Love wave reduces into

$$
\begin{aligned}
& \tan \left[k H \sqrt{\left.\left(\frac{\alpha}{2 k}\right)^{2}+\frac{c^{2}}{c^{2}{ }_{1}}+\frac{P_{0}-2 a_{3}}{2 a_{1}}\right]}\right. \\
& =\frac{\left[\left(\frac{\alpha}{2 k}\right) \frac{\cos (\alpha \mathrm{H})}{1-\sin (\alpha \mathrm{H})}-\left\{\frac{\mu_{2}}{a_{1}}\left(-1+\frac{1}{2} \frac{c^{2}}{c^{2}}\right)+\frac{\alpha}{2 k}\right\}\right] \sqrt{\left(\frac{\alpha}{2 k}\right)^{2}+\frac{c^{2}}{c^{2}}+\frac{P_{0}-2 a_{3}}{2 a_{1}}}}{\left(\frac{\alpha}{2 k}\right)^{2}+\frac{c^{2}}{c^{2}}+\frac{P_{0}-2 a_{3}}{2 a_{1}}+\left(\frac{\alpha}{2 k}\right) \frac{\cos (\alpha \mathrm{H})}{1-\sin (\alpha \mathrm{H})}\left\{\frac{\mu_{2}}{a_{1}}\left(-1+\frac{1}{2} \frac{c^{2}}{c^{2}{ }_{2}}\right)+\frac{\alpha}{2 k}\right\}}
\end{aligned}
$$

Case VII: If $\alpha \rightarrow 0, a_{1}=a_{3}=u_{1}, P_{0}=0, d \rightarrow$ $1, N=L=u_{2}, G=0$, i.e., the upper layer is homogeneous, isotropic and free from initial stress and the layer is non-porous, isotropic and free from gravity, the dispersion equation (5.7) takes the form

$$
\tan \left[k H \sqrt{\frac{c^{2}}{c^{2}}-1}\right]=\frac{\mu_{2}}{\mu_{1}} \frac{\sqrt{1-\frac{c^{2}}{c^{2}}}}{\sqrt{\frac{c^{2}}{c^{2}}-1}}
$$

which is the well-known classical result of Love wave (Love, 1927) in an orthotropic homogeneous layer over a homogeneous isotropic half-space and hence validating the solution of the problem discussed.

\section{Numerical Results and Discussion}

Taking a particular model, the propagation of Love waves in a heterogeneous orthotropic layer under changeable initial stress over a gravitating porous halfspace is discussed. In order to show the effect of $\frac{\alpha}{2 k}, \quad \mathrm{I}=\frac{2 a_{1}}{2 a_{3}-P_{0}}, \frac{N}{L}, \frac{L}{a_{1}}, \frac{c_{1}}{c_{2}}, d$ and Biot's gravity parameter $G$ on the propagation of Love wave, the curves have been plotted for the dispersion equation (5.7). In all the figures, curves have been plotted with vertical axis as dimensionless phase velocity $\frac{c}{c_{1}}$ against horizontal axis as dimensionless wave number $k H$. It has been noticed that the phase velocity decreases rapidly for the increasing value of wave number in each of the figures under the considered values of various parameters. The results are presented in Figs. 2 to 8.

Table 1: Values of dimensionless parameters selected for various plots of the dispersion equation

$\begin{array}{lllllll}\text { Fig. } 2 & \text { Fig. } 3 & \text { Fig. } 4 & \text { Fig. } 5 & \text { Fig. } 6 & \text { Fig. } 7 & \text { Fig. } 8\end{array}$

\begin{tabular}{lccccccc}
$\frac{\alpha}{2 k}$ & - & 0.2 & 0.2 & 0.2 & 0.2 & 0.2 & 0.2 \\
$\mathrm{I}_{c_{1}}$ & 1.4 & - & 1.4 & 1.4 & 1.4 & 1.4 & 1.4 \\
$\frac{c_{2}}{N}$ & 0.75 & 0.75 & - & 0.75 & 0.75 & 0.75 & 0.75 \\
$\frac{N}{L}$ & 0.6 & 0.6 & 0.6 & - & 0.6 & 0.6 & 0.6 \\
$\frac{L}{a_{1}}$ & 0.2 & 0.2 & 0.2 & 0.2 & - & 0.2 & 0.2 \\
$d$ & 0.6 & 0.6 & 0.6 & 0.6 & 0.6 & - & 0.6 \\
$G$ & 0.6 & 0.6 & 0.6 & 0.6 & 0.6 & 0.6 & - \\
\hline
\end{tabular}


Fig. 2 shows the effect of heterogeneity parameter $\frac{\alpha}{2 k}$ on the propagation of Love waves. The value of $\frac{\alpha}{2 k}$ has been considered as 0.0, 0.3, 0.6 and 0.9 for curves $1,2,3$ and 4 respectively. It is observed that as the heterogeneity increases, the phase velocity of Love wave increases. Here the curves are merging at a single point, thereby revealing that even if $\frac{\alpha}{2 k}$ varies, phase velocity remains constant for the same frequency.

Fig. 3 displays the effect of initial compression $I$ on the propagation of Love wave in a heterogeneous orthotropic layer overlying a gravitating porous halfspace. The value of $I$ for curves 1, 2, 3 and 4 has been taken as 1.2, 1.4, 1.6 and 1.8 respectively. We observe that the effect of initial compression is very prominent on the propagation of Love wave. The increase of initial compression of the layer can decrease the magnitude of phase velocity, i.e., the presence of initial stress of the layer reduces the velocity. The curves are also little far apart from each other at lower phase velocity which reveals that the parameter $I$ has significant dominance for high values of wave number.

Fig. 4 shows the dispersion curves for different values of $\frac{c_{1}}{c_{2}}$. The value of $\frac{c_{1}}{c_{2}}$ for curves $1,2,3$ and 4 has been taken as $0.75,0.80,0.85$ and 0.90 respectively. The figure shows that as the value of $\frac{c_{1}}{c_{2}}$ increases, the phase velocity of Love wave decreases. It has also been noticed that prominent effect of this parameter is visible only at lower frequency.

In Fig. 5, the effect of anisotropic factor $\frac{N}{L}$ has been shown. For curves 1, 2, 3 and 4 the value of $\frac{N}{L}$ has been taken as $0.5,0.6,0.7$ and 0.8 respectively.
From this figure it has been observed that as the dimensionless ratio $\frac{N}{L}$ increases the phase velocity also increases at the same frequency.

Fig. 6 gives the dispersion curves for different values of $\frac{L}{a_{1}}$. The value of this ratio for curves 1,2 , 3 and 4 has been taken as $0.2,0.3,0.4$ and 0.5 respectively. This figure shows that this dimensionless parameter has remarkable effect on the phase velocity. For fixed wave number, phase velocity increases with increasing $\frac{L}{a_{1}}$. It has also been observed that the velocity graph tends to merge at higher phase velocity and lower frequency.

Fig. 7 shows the effect of porosity on the phase velocity. The value of $d$ for curves 1, 2, 3 and 4 has been taken as $0.0,0.2,0.4$ and 0.6 respectively. It shows that as the porosity increases, the phase velocity of Love waves decreases.

Fig. 8 shows that the effect of gravity on the propagation of Love wave is also significant. In the figure, the value of Biot's gravity parameter $G$ for curves 1,2, 3 and 4 has been considered as 0.40 , $0.55,0.70$ and 0.85 respectively. As the gravity of half-space increases, the phase velocity of Love waves decreases.

\section{Conclusions}

The present study is helpful to find out the wave velocity of Love waves in a heterogeneous orthotropic layer under changeable initial stress over a gravitating porous half-space. Dispersion equation (5.7) for some special cases of interest has been derived. When the layer is homogeneous, isotropic and free from initial stress and the half-space is also isotropic, non-porous and free from gravity, the dispersion equation reduces into a classical result of Love wave. Phase velocity is computed numerically and the effects of variation in $\frac{L}{a_{1}}, \frac{c_{1}}{c_{2}}$, ratio of directional rigidities, $\frac{N}{L}$, porosity, d, 


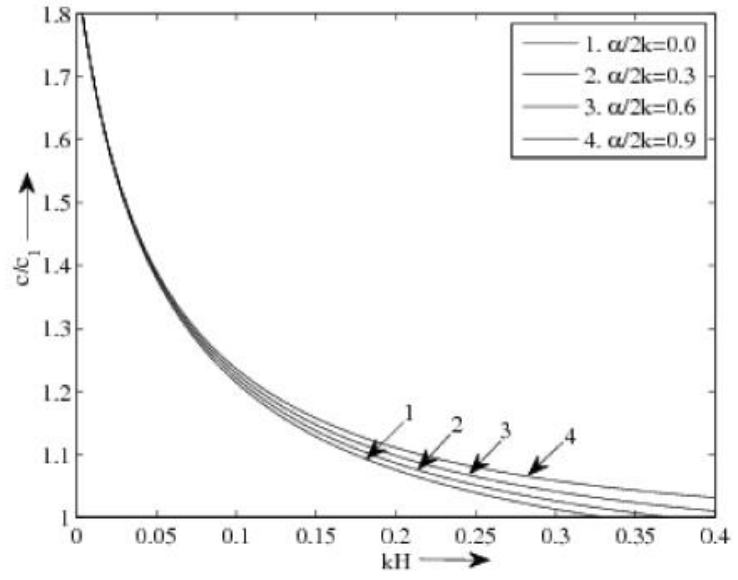

Fig. 2: Dimensionless phase velocity as function of dimensionless wave number of Love waves for different values of $\frac{\alpha}{2 k}$

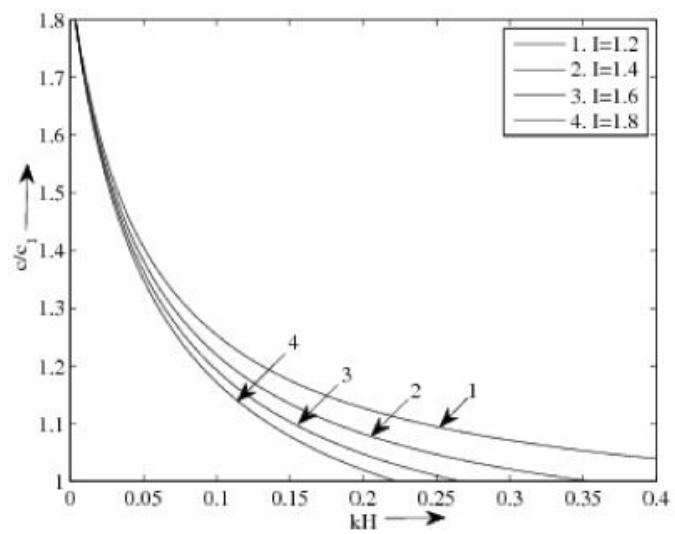

Fig. 3: Dimensionless phase velocity as function of dimensionless wave number of Love waves for different values of $I$

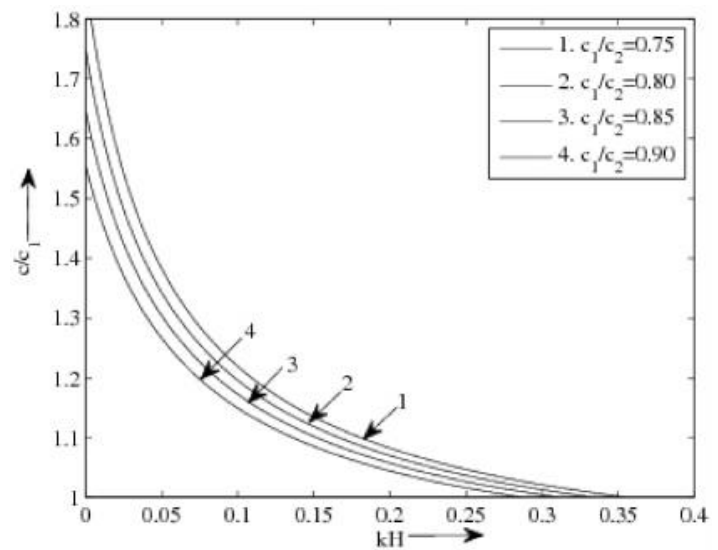

Fig. 4: Dimensionless phase velocity as function of dimensionless wave number of Love waves for different values of $\frac{c_{1}}{c_{2}}$

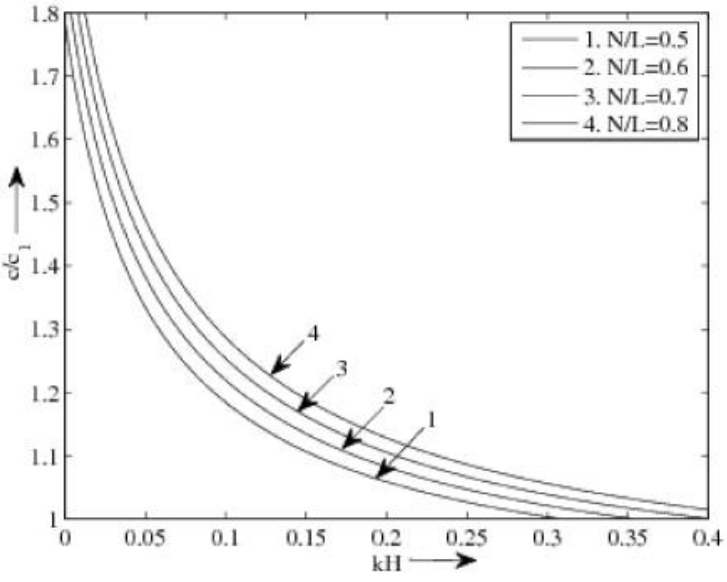

Fig. 5: Dimensionless phase velocity as function of dimensionless wave number of Love waves for different values of $\frac{N}{L}$

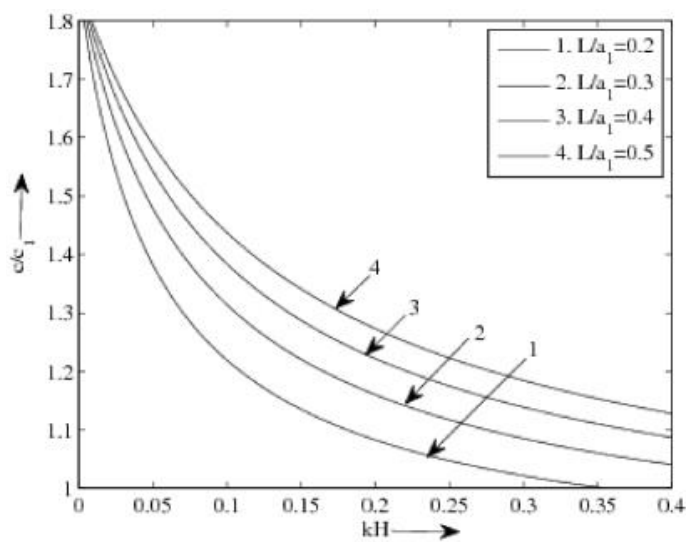

Fig. 6: Dimensionless phase velocity as function of dimensionless wave number of Love waves for different values of $\frac{L}{a_{1}}$

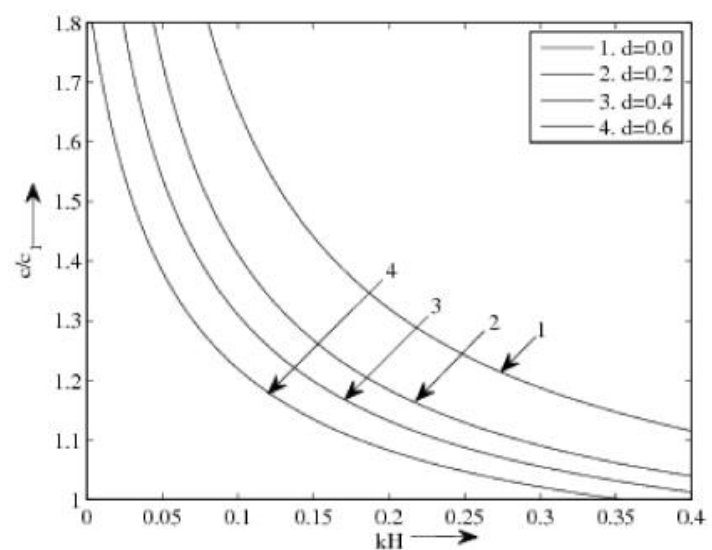

Fig. 7: Dimensionless phase velocity as function of dimensionless wave number of Love waves for different values of $d$ 


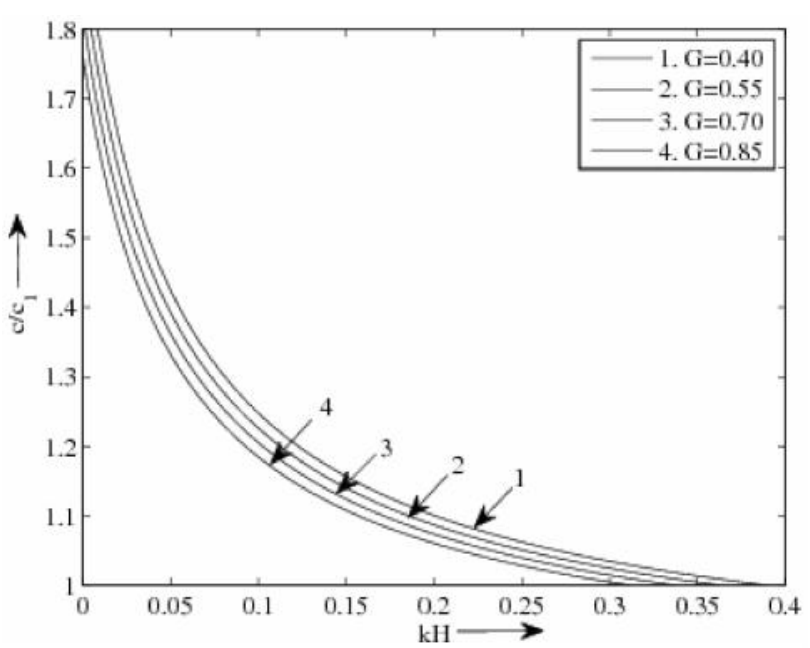

Fig. 8: Dimensionless phase velocity as function of dimensionless wave number of Love waves for different values of $G$

Biot's gravity parameter, $G$ and heterogeneity parameter, $\frac{\alpha}{2 k}$ are shown graphically. For graphical representation, MATLAB software has been used. We have observed that

(i) Dimensionless phase velocity $\frac{c}{c_{1}}$ of Love wave increases with the decrease of dimensionless wave number $k H$ in all the figures.

\section{References}

Abd-Alla A M, Abo-Dahab S M and Al-Thamali T A (2013a) Love waves in a non-homogeneous orthotropic magnetoelastic layer under initial stress overlying a semiinfinite medium in Journal of Computational and Theoretical Nanoscience 10 10-18

Abd-Alla A M, Abo-Dahab S M and Al-Thamali T A (2012) Propagation of Rayleigh waves in a rotating orthotropic material elastic half-space under initial stress and gravity in Journal of Mechanical Science and Technology 26 2815-2823

Abd-Alla A M and Ahmed S M (1999) Propagation of Love waves in a non-homogeneous orthotropic elastic layer (ii) The presence of initial compression reduces the velocity of Love wave.

(iii) The phase velocity of Love wave increases with increasing $\frac{L}{a_{1}}, \frac{N}{L}$ and heterogeneity parameter $\frac{\alpha}{2 k}$

(iv) The effects of porosity and Biot's gravity parameter are very prominent on the propagation of Love waves. The higher the value of porosity, $\mathrm{d}$ or gravity parameter, $\mathrm{G}$ lower is the phase velocity.

Since the phase velocity is affected by various dimensionless parameters, the present study will be useful for the study of seismic waves generated by artificial explosion and it may provide valuable information about the selection of proper structural materials for present day construction work. The results presented in this paper may be useful for researchers of material science and designers of new materials.

\section{Acknowledgements}

The authors convey their sincere thanks to Indian School of Mines, Dhanbad-826004, India, for providing JRF to Mr. Anup Saha and also facilitating us with the best facilities.

under initial stress overlying semi-infinite medium in Applied Mathematics and Computation 106 265-275

Abd-Alla A M, Nofal T A, Abo-Dahab S M and Al-Mullise A (2013b) Surface waves propagation in fibrereinforced anisotropic elastic media subjected to gravity field in International Journal of Physical Sciences 8 574584

Ahmed S M and Abo-Dahab S M (2010) Propagation of Love waves in an orthotropic Granular layer under initial stress overlying a semi-infinite Granular medium inJournal of Vibration of Control 16 1845-1858

Biot M A (1956) Theory of propagation of elastic waves in a fluid-saturated porous solid.I. Low-frequency range in 
Journal of the Acoustical Society of America 28 168-178

Biot M A (1965) Mechanics of Incremental Deformation.John Willey and Sons

Chattaraj R and Samal S K (2013a) Love waves in the fibrereinforced layer over a gravitating porous half-space in Acta Geophysica 61 1170-1183

Chattaraj R, Samal S K and Mahanti N C (2013b) Dispersion of Love wave propagating in irregular anisotropic porous stratum under initial stress in International Journal of Geomechanics 13 402-408

Chattopadhyay A and De R K (1983) Love type waves in a porous layer with irregular interface in International Journal of Engineering Science 21 1295-1303

Dey S and Dutta D (1992) Torsional wave propagation in an initially stressed cylinder in Proceedings of the Indian National Science Academy 58 425-429

Dey S, Gupta A K and Gupta S (1998) Propagation of torsional surface waves in dry sandy medium under gravity in Mathematics and Mechanics of Solid 3 229-235

Dey S and Gupta S (1987) Longitudinal and shear waves in an elastic medium with void pores in Proceedings of the Indian National Science Academy 53 554-563

Ewing W M, Jardetzky W S and Press F (1957) Elastic waves in layered media. McGraw-Hill

Ghorai A P, Samal S K and Mahanty N C (2010) Love waves in a fluid saturated porous layer under a rigid boundary and lying over an elastic half-space under gravity inApplied Mathematical Modelling 34 1873-1883

Gupta S, Majhi D K, Kundu S and Vishwakarma S K (2013a) Propagation of Love waves in a non-homogeneous substratum over initially stressed heterogeneous halfspace inApplied Mathematics and Mechanics 34 249-258

Gupta S, Vishwakarma S K, Majhi D K and Kundu S (2013b) Possibility of Love wave propagation in a porous layer under the effect of linearly varying directional rigidities in Applied Mathematical Modelling 37 6652-6660

Kundu S, Gupta S, Chattopadhyay A and Majhi D K (2013) Love wave propagation in porous rigid layer lying over an initially stressed half-space inInternational Journal of Applied Physics and Mathematics $\mathbf{3}$

Kundu S, Gupta S and Manna S (2014a) Propagation of Love wave in fibre-reinforced medium lying over an initially stressed orthotropic half-space inInternational Journal for Numerical and Analytical Methods in Geomechanics 38 1172-1182

Kundu S, Manna S and Gupta S (2014b) Love wave dispersion in pre-stressed homogeneous medium over a porous halfspace with irregular boundary surfaces in International Journal of Solid and Structures 51 3689-3697

Love A E H (1927) The mathematical theory of elasticity. Cambridge University Press. Manna S, Kundu S and Gupta S (2013) Love wave propagation in a piezoelectric layer overlying in an inhomogeneous elastic half-space inournal of Vibration of Control doi: 10.1177/1077546313513626

Midya G K (2004) On Love-type surface waves in homogeneous micropolar elastic media in International Journal of Engineering Science 42 1275-1288

Whittaker E T and Watson G N (1990) A course in modern analysis Cambridge University Press. 